\title{
Quantitative Determination of Morphometric Indices of the Total and Perfused Capillary Network of the Newborn Pig Brain
}

\author{
MUJAHID ANWAR, JOHANNA WEISS, AND HARVEY R. WEISS \\ Departments of Pediatrics [M.A.] and Physiology and Biophysics [J.W., H.R.W.], Heart and Brain Circulation \\ Laboratory, University of Medicine and Dentistry of New Jersey, Piscataway, New Jersey 08854-5635
}

\begin{abstract}
The purpose of this study was to determine if the newborn pig brain had a reserve of unperfused capillaries during normoxia. To accomplish this, a method was developed to determine the volume fraction, surface area, and number of both total and perfused capillaries in the newborn pig brain. Newborn pigs of either sex, 2-7 d old, were used. FITC-dextran, molecular weight 147000 , was used as a plasma marker to visualize the perfused capillaries. Alkaline phosphatase staining was used to stain the total capillary bed of the brain. Our results showed that FITC-dextran stayed within the vascular compartment, as it was not seen in areas that were not subsequently visualized with alkaline phosphatase staining. Eighty-four to $86 \%$ of the alkaline phosphatase-stained capillaries could be visualized with perfusion markers (india ink or FITC-dextran) in different brain regions. Similar results were obtained in two animals using a basement membrane stain, silver methenamine. The total volume fraction of capillaries $\left(\mathrm{mm}^{3} / \mathrm{mm}^{3}\right)$ was cortex $0.055 \pm 0.012$, cerebellum $0.062 \pm 0.011$, and medulla $0.039 \pm 0.012$. Capillary surface area $\left(\mathrm{mm}^{2} / \mathrm{mm}^{3}\right)$ of different brain regions averaged cortex $23.2 \pm 1.8$, cerebellum $24.8 \pm 2.5$, and medulla 15.8 \pm 2.9 . The total number of capillaries $\left(\right.$ per $\mathrm{mm}^{2}$ ) was cortex $375 \pm 37$, cerebellum $329 \pm 37$, and medulla $216 \pm 32$. The time course of filling of the capillaries indicated that approximately $50 \%$ were perfused at $6 \mathrm{~s}$, which increased to over $80 \%$ at $12 \mathrm{~s}$ and remained unchanged thereafter. We found that FITC-dextran (molecular weight 147000 ) was a suitable plasma marker to visualize the perfused capillaries, and alkaline phosphatase staining could mark the total capillary bed in the newborn pig brain. We conclude that the capillaries in the newborn pig brain fill rapidly and that this organ lacks a capillary reserve at rest. (Pediatr Res 32: 542-546, 1992)
\end{abstract}

Oxygen supply to the tissues is determined by the blood flow and oxygen content of the blood as well as the diffusion distances for oxygen from the capillaries to the neurons. Cerebral blood flow has been studied extensively in both adult and newborn animals $(1,2)$. In general, cerebral blood flow is closely linked to metabolism. In mature animals, the number of perfused cerebral capillaries may also increase under some conditions (3). In the newborn, blood pressures tend to be lower than in adults, but they have cerebral autoregulatory ability (4). To maintain cerebral blood flow, the newborn cerebral vasculature has a lower

Received February 11, 1992; accepted July 14, 1992

Correspondence and reprint requests: Harvey R. Weiss, Ph.D., Department of Physiology \& Biophysics, Heart and Brain Circulation Laboratory, UMDNJ-Robert Wood Johnson Medical School, 675 Hoes Lane, Piscataway, NJ 08854-5635.

Supported in part by USPHS Grant NS25100. resistance than adults. Any condition that results in decreased oxygen supply or increased metabolic needs could cause increased blood flow or decreased diffusion distance.

In newborn animals, cerebral capillarity tends to be lower than in adults in those animals with incomplete development, whereas species born more completely developed tend to have better capillary development $(5-7)$. Newborn pigs tend to have a better developed cerebral vasculature than newborn rats $(4,7)$. It was not known whether all of these cerebral capillaries were perfused in normoxia.

The purpose of this study was to test the hypothesis that all cerebral capillaries were perfused during normoxia in the newborn pig. Diffusion distance in tissue is determined by the number of perfused capillaries at any given time in that tissue. Weiss et al. (3) have developed a method in the adult rat to study both the perfused and total capillaries in the same brain section using FITC-dextran to label perfused capillaries and an alkaline phosphatase stain for the total capillary bed. Thus, the capillary reserve at rest as well as use of that reserve under various conditions can be studied. In a number of organs examined, including the brain, not all capillaries are perfused at all times. In the adult rat, this capillary reserve in the brain can be mobilized during hypoxia, asphyxia, and hypotension $(3,8,9)$. The perfusion of cerebral capillaries has not been studied in newborn animals. In the present study, we adapted the method of Weiss et al. (3) for labeling total and perfused cerebral capillaries to the newborn pig to ascertain if the newborn pig has a capillary reserve at rest. In particular, it was not known if plasma markers like FITC-dextran would stay within the vascular compartment and not leak into the brain parenchyma. In addition, it was not known if alkaline phosphatase was present in sufficient quantity to reliably stain the capillary endothelium. We determined that the capillaries in the newborn pig brain fill rapidly and that this organ lacks a capillary reserve at rest.

\section{MATERIALS AND METHODS}

Newborn pigs 2 to $7 \mathrm{~d}$ old of either sex were used. Their average weight was $1.9 \pm 0.5 \mathrm{~kg}$. Anesthesia was induced with ketamine $30 \mathrm{mg} / \mathrm{kg}$ and xylazine $10 \mathrm{mg}$ intramuscularly. Additional ketamine in $10-\mathrm{mg} / \mathrm{kg}$ aliquots was used up to $60 \mathrm{mg} / \mathrm{kg}$ total dose to maintain adequate anesthesia for the insertion of a femoral venous line. After the establishment of the venous line, anesthesia was maintained with $\alpha$-chloralose $40 \mathrm{mg} / \mathrm{kg}$ in four divided doses as needed. A femoral arterial line was inserted for the monitoring of arterial blood pressure [R411 recorder (Beckman Instruments, Inc., Fullerton, CA) and $\mathrm{P} 23 \mathrm{Db}$ transducer (Statham, Cambridge, MA)] and blood gases (ABL 330, Radiometer, Copenhagen, Denmark). Body temperature was maintained at $37^{\circ} \mathrm{C}$ with a rectal thermistor and a heat lamp. Animals were allowed to stabilize for half an hour before the measurements of total and perfused capillaries were performed. 
After the final determination of heart rate, blood pressure, and arterial blood gases, $150 \mathrm{mg} / \mathrm{kg}$ FITC-dextran (molecular weight 147000 ) was injected rapidly i.v. as a $1.5-\mathrm{mL}$ bolus. To determine the time course of filling of the capillaries, the FITCdextran was allowed to circulate for a varying time period from $6 \mathrm{~s}$ to $6 \mathrm{~min}$, at which time the animals were decapitated. Brains were quickly removed and frozen at $-70^{\circ} \mathrm{C}$ until analyzed. The following brain regions were isolated and prepared for cutting on a microtome-cryostat set at $-35^{\circ} \mathrm{C}$ : cortex, cerebellum, and medulla. The tissue sections were mounted on a microtome specimen holder and coated with embedding medium for frozen tissue specimens. Three to $5-\mu \mathrm{m}$ thick sections were cut. The sections were transferred to previously marked glass slides gently scratched with a diamond point. The sections were allowed to dry at room temperature in the dark for $2 \mathrm{~h}$. Between 10 and 12 sections were obtained for each brain region, each section being at least $200 \mu \mathrm{m}$ from the previous one.

The slides were then photographed on a Zeiss fluorescent microscope equipped for automated photography. A $40 \times$ objective was used to photograph the capillaries. The slides were epiilluminated with UV light from a $100-W$ halogen source to excite the fluorescence in the FITC-dextran. A barrier filter was placed in the viewing field to allow $495-\mu \mathrm{m}$ or greater wavelength light through. A second photograph of the same field was taken in the normal light, which, together with the viewing coordinates obtained, helped relocate the field.

The slides were then stained for alkaline phosphatase. The slides were fixed in a sucrose-formalin buffer for $1 \mathrm{~min}$, washed twice in distilled water, and then placed in an incubation mixture for $8 \mathrm{~min}$ at $37^{\circ} \mathrm{C}$. The incubation mixture consisted of $3.8 \mathrm{~g} / \mathrm{L}$ fast blue RR, $0.5 \mathrm{~g} / \mathrm{L} \alpha$-naphthyl phosphate, $3.8 \mathrm{~g} / \mathrm{L}$ sodium metaborate, and $1.7 \mathrm{~g} / \mathrm{L}$ magnesium sulfate. The slides were postfixed and dried.

Various stereologic determinations were performed counting each field twice, once for the total and once for the perfused capillaries. These stereologic procedures have been reviewed by Weibel (10) and applied to the brain microvasculature by Weiss et al. (3). A Dapple image analyzing device was used. Slides stained for alkaline phosphatase were placed under a microscope, relocating the field previously photographed for fluorescence. The image analyzer was used to evaluate the slides for total and then for perfused morphometric indices of the capillaries. The image was obtained from a Panasonic TV camera attached to the microscope and digitized with the Dapple image analyzer. The program digitizes the raw image by measuring the brightness in 64 gray scale level steps at each point in a $254 \times 192$ array in the picture. The image is edited through the various subroutines in the Dapple system and manually edited with a light pen.

The volume fraction (in $\mathrm{mm}^{3} / \mathrm{mm}^{3}$ ) of the capillaries was determined by dividing the number of test points falling within the profile of a capillary by the total number of points within the array. The total number of test points were selected such that the probable error in volume fraction would be less than $\pm 5 \%$. The number of capillaries $/ \mathrm{mm}^{2}$ was determined from the number of vessels per unit test area. The surface area (in $\mathrm{mm}^{2} / \mathrm{mm}^{3}$ ) was also determined. After the total capillaries were studied, the perfused portion was obtained from photographs of the fluorescence within the capillaries. The light pen was used to edit the digitized image for measurements of the perfused capillaries.

To assess if alkaline phosphatase staining and/or plasma markers would visualize all the capillaries in the brain, three different methods were used. In three animals, cerebral circulation was perfused with india ink after performing a thoracotomy and washing out the blood from the circulation with saline. Slides were prepared and photographed as described above to visualize the india ink-filled capillaries using ordinary light. Slides were then stained for alkaline phosphatase, and the same sections that were photographed were compared to see if the capillaries that were filled with india ink could be visualized with alkaline phosphatase staining. In another three animals, FITC-dextran was allowed to circulate for $6 \mathrm{~min}$, a time period in which in the adult rats almost $100 \%$ of the capillaries are filled with FITCdextran, and similar comparison was made of the FITC-dextran visualized capillaries and the alkaline phosphatase-stained capillaries. In another two rats that were perfused with FITC-dextran for a prolonged period, cerebral cortical capillaries were visualized with silver methenamine, a basement membrane stain. The ratio of the number of capillaries filled with FITC-dextran to the total number of capillaries visualized with silver methenamine was compared with that using alkaline phosphatase. Eight to 10 sections from each brain region were used for these comparisons.

The data are expressed as mean \pm SD. Differences between regions and between different groups of animals were assessed using a factorial analysis of variance. A Duncan post hoc procedure was used when the analysis of variance indicated significant differences. A $p$ value $<0.05$ was considered significant. The study was approved by the Robert Wood Johnson Medical School Institutional Animal Care and Use Committee, and animals were handled according to the guidelines established by the National Institutes of Health for care and use of laboratory animals.

\section{RESULTS}

All animals were hemodynamically stable at the time of decapitation for morphometric measurements. They had an average heart rate of $114 \pm 24$ beats/min and a systolic and diastolic blood pressure of $10.9 \pm 1.6 \mathrm{kPa}(82 \pm 12 \mathrm{~mm} \mathrm{Hg})$ and $6.3 \pm$ $0.8(47 \pm 6)$, respectively. Arterial blood gases were also in the normal range, with a $\mathrm{PO}_{2}$ of $12.2 \pm 2.0 \mathrm{kPa}(92 \pm 15 \mathrm{~mm} \mathrm{Hg})$, a $\mathrm{PCO}_{2}$ of $4.8 \pm 0.4 \mathrm{kPa}(36 \pm 3 \mathrm{~mm} \mathrm{Hg})$, and a pH of $7.41 \pm$ 0.07 .

Table 1 shows the total volume fraction, surface area, and number of capillaries in three different examined brain regions. These data were obtained from the alkaline phosphatase-stained brain sections. In general, different brain regions were quite similar with respect to the variables studied. However, there were some significant differences. The volume fraction of the total capillaries was higher in cerebellum in comparison to other brain regions, and the number of capillaries was lower in the medulla in comparison to the cortex.

Figure 1 shows an example of the cortical capillary network stained for alkaline phosphatase. A second photograph of the fluorescence of FITC-dextran filling of the field shows good correspondence. Figures $2 A-C$ show the time course of visualization of the cerebral capillaries in the various brain regions after i.v. injection of FITC-dextran. At $6 \mathrm{~s}$ after injection, approximately $50-60 \%$ of the capillaries were filled with FITC-dextran. This value increased to $80-85 \%$ at the $12-\mathrm{s}$ time point. There were no further changes in the percentage perfused thereafter until $6 \mathrm{~min}$. Each point represents the mean \pm SD for three to five animals. The time course of filling of the capillary bed was similar in all regions.

To verify that we were labeling both the perfused and total capillary network, validation studies were conducted. The percentage of the capillary volume fraction stained with alkaline phosphatase, which was visualized with india ink, or FITCdextran circulated for 6 min was determined. The percentage of perfused cerebral capillaries that were stained by silver methe-

Table 1. Total volume fraction $\left(V_{v}, \mathrm{~mm}^{3} / \mathrm{mm}^{3}\right)$, surface area $\left(S_{v}, \mathrm{~mm}^{2} / \mathrm{mm}^{3}\right)$, and number $\left(N_{a}\right.$, no. $\left./ \mathrm{mm}^{2}\right)$ of capillaries in different brain regions

\begin{tabular}{llll}
\hline & \multicolumn{1}{c}{$\mathrm{V}_{\mathrm{v}}$} & \multicolumn{1}{c}{$\mathrm{S}_{\mathrm{v}}$} & \multicolumn{1}{c}{$\mathrm{N}_{\mathrm{a}}$} \\
\hline Cortex $(n=18)$ & $0.0462 \pm 0.00098$ & $20.7 \pm 3.7$ & $374 \pm 82$ \\
Cerebellum $(n=17)$ & $0.0488 \pm 0.0143^{*}$ & $21.3 \pm 4.1$ & $313 \pm 53$ \\
Medulla $(n=15)$ & $0.0420 \pm 0.0109$ & $18.0 \pm 4.4$ & $279 \pm 101 \dagger$ \\
\hline
\end{tabular}

* Significantly higher than other brain regions.

$\dagger$ Significantly lower than cortex. 

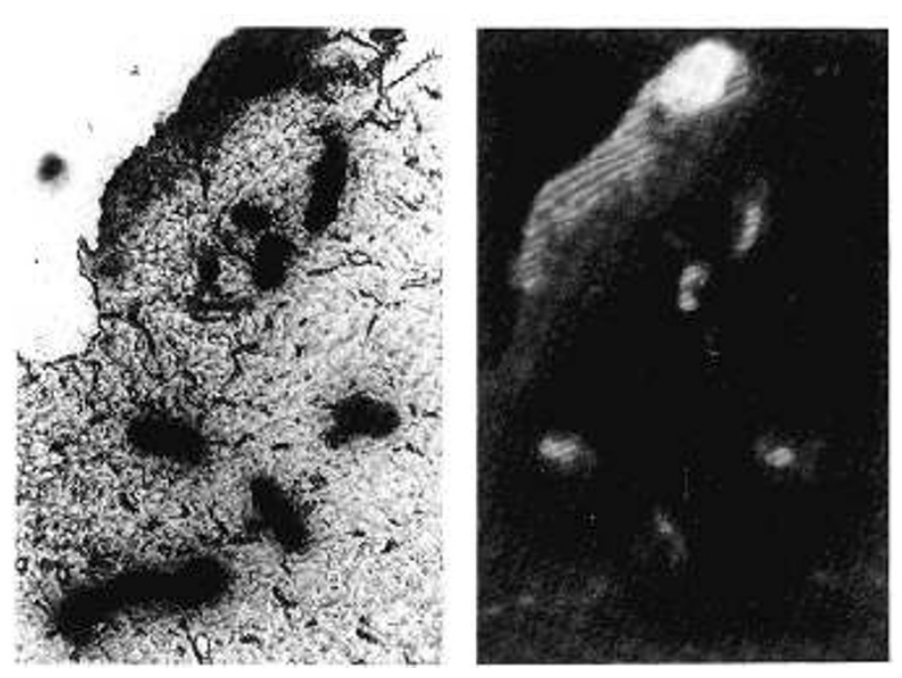

Fig. 1. On the left side of the figure, the dark areas represent alkaline phosphatase-stained microvessels from the cerebral cortex of a newborn pig. On the right, the light areas show the presence of FITC-dextran in these microvessels. The FITC-dextran circulated for $20 \mathrm{~s}$. Note the good correspondence between the total and perfused capillary network, indicating no reserve of unperfused vessels. Bar $=10 \mu \mathrm{m}$.

namine was also determined. Similar numbers of capillaries were visualized with silver methenamine and alkaline phosphatase. Of the capillaries stained for alkaline phosphatase, $84 \pm 2 \%$ of cortical and $86 \pm 10 \%$ of cerebellar capillary volume fraction was visualized by india ink. FITC-dextran circulated for $6 \mathrm{~min}$ visualized $85 \pm 2,86 \pm 1$, and $88 \pm 7 \%$ of cortical, cerebellar, and medullary capillary volume fractions, respectively. We also found that $84 \pm 6 \%$ of cortical capillaries stained with silver methenamine were labeled with FITC-dextran. There were no capillaries filled with india ink or FITC-dextran that were not subsequently visualized with alkaline phosphatase or silver methenamine staining. Two additional animals were asphyxiated for 1.5-2 min. At this time, blood pressure was $11.6 \pm 2.3 / 6.9$ $\pm 0.5 \mathrm{kPa}(87 \pm 17 / 52 \pm 4 \mathrm{~mm} \mathrm{Hg})$, heart rate was $102 \pm 17$ beats/min, arterial $\mathrm{PO}_{2}$ was $3.7 \pm 3.5 \mathrm{kPa}(28 \pm 26 \mathrm{~mm} \mathrm{Hg})$, arterial $\mathrm{PCO}_{2}$ was $6.0 \pm 0.5 \mathrm{kPa}(45 \pm 4 \mathrm{~mm} \mathrm{Hg})$, and pH 7.26 \pm 0.04 . When FITC-dextran circulated for $12 \mathrm{~s}$, we found $68 \pm$ $2,79 \pm 7$, and $67 \pm 5 \%$ of cortical, cerebellar, and medullary capillary volume fraction perfused.

\section{DISCUSSION}

The main conclusion of our study was that the newborn pig lacks a usable cerebral capillary reserve. The capillary bed of the newborn pig brain fills rapidly with a plasma marker (FITCdextran), unlike that of adult rats (3). In normoxia, the pig does not possess the ability to reduce its cerebral diffusion distance. Thus, although cerebral blood flow can increase in response to increased demand, cerebral diffusion distance for oxygen is minimal at rest in the newborn pig.

This conclusion depends on our ability to visualize both the total and perfused cerebral capillary network in the newborn pig. We used an alkaline phosphatase stain to study the total capillary bed. Alkaline phosphatase is expressed in capillary endothelial cells, but in developing mouse or rat this expression is reduced (11). The newborn pig has a more mature cerebral vasculature, and we found alkaline phosphatase activity to be quite high. We had to reduce the staining time significantly in the pig compared with the adult rat (3). However, we also compared this marker to a basement membrane stain, silver methenamine, and found similar values. In comparison to plasma markers such as FITCdextran and india ink, we found about $15 \%$ more alkaline phosphatase-stained vessels. Mature pigs also have good alkaline phosphatase activity in cerebral microvessels (12).
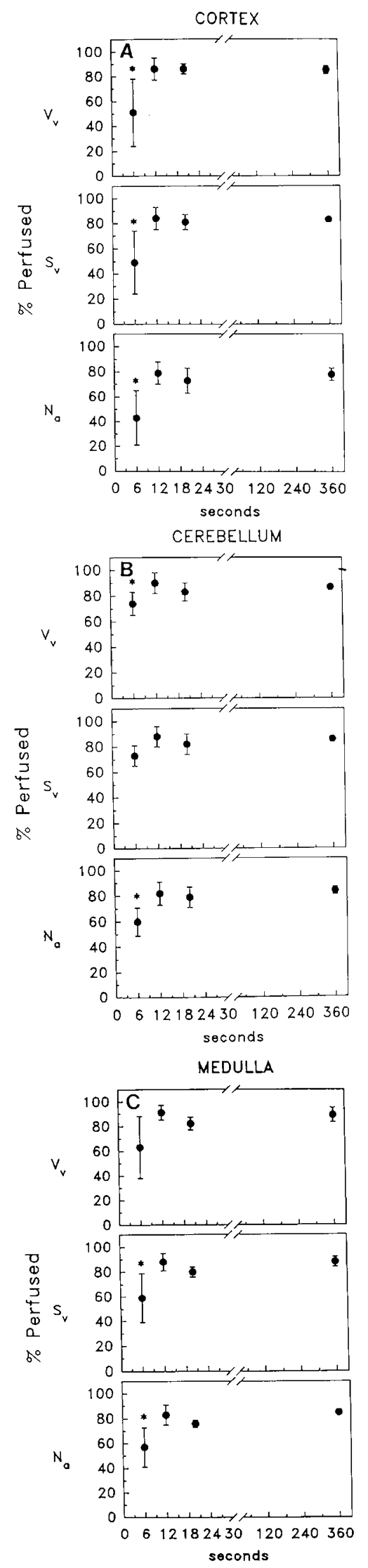

Fig. 2. The percentage of the cortical $(A)$, cerebellar $(B)$, and medullary $(C)$ capillaries labeled with FITC-dextran at various time points after injection. The percentage of the capillary volume fraction $\left(V_{v}\right)$ surface area per unit volume $\left(S_{v}\right)$, and number $/ \mathrm{mm}^{2}\left(N_{a}\right)$ perfused are shown. 
Our data show that FITC-dextran (molecular weight 147 000) can visualize the perfused cerebral capillaries in the newborn pig. We did not see leakage of FITC-dextran outside the capillaries; under no circumstance did we see fluorescent spots in an area of tissue that was not subsequently stained for alkaline phosphatase. Weiss and coworkers $(3,8,9)$ have used FITC-dextran (molecular weight 70000 ) for their studies on cerebral circulation in the adult rat. Because newborn animals are considered to have a more leaky blood-brain barrier than adults, we chose a larger molecular weight dextran for these studies. In the newborn rat, the transfer of substance across the blood-brain barrier is considerably faster than in adult rat (13). However, in newborn rabbit and pig, the blood-brain barrier appears to be quite mature and similar to the adult barrier $(14,15)$.

There is some controversy about the ability of the technique used in this study to visualize both the total and perfused cerebral capillary bed in the adult rat. Weiss and coworkers $(3,8,9,16)$ have presented data to indicate that under resting conditions approximately $50 \%$ of the capillary bed that can be visualized with alkaline phosphatase was filled with FITC-dextran that was allowed to circulate for $20 \mathrm{~s}$. However, when the animals were asphyxiated or if the FITC-dextran was allowed to circulate for $6 \mathrm{~min}$, all the capillary bed visualized with alkaline phosphatase was perfused with FITC-dextran. In contrast, the method used by Gobel et al. and Klein et al. (17-19) consistently shows a lack of capillary reserve in the adult rat. Several differences in the methods used by Weiss et al. and those used by Gobel et al. and Klein et al. can account for these discrepant results (16). Because of this controversy, we used several methods of visualizing the total and perfused cerebral capillary bed to be certain that our results were accurate in the newborn pig. With perfusion markers (india ink and FITC-dextran) circulated for more than $12 \mathrm{~s}$, we could demonstrate that $84-88 \%$ of the capillaries were filled compared with alkaline phosphatase stain. In two animals (1012 slides from each animal), silver methenamine, a basement membrane stain, was compared with the FITC-dextran-perfused capillaries. Of the capillaries stained with silver methenamine, $84 \%$ were visualized with FITC-dextran, a number similar to that with alkaline phosphatase. Asphyxia did not fill all capillaries with FITC-dextran. In addition, we never saw our perfused marker outside a capillary stained with alkaline phosphatase. Thus, alkaline phosphatase can reliably stain the total capillary bed and FITC-dextran can reliably stain the perfused capillaries of the newborn pig.

The average total volume fraction of capillaries in our study was $4.2-4.9 \%$ of the tissue volume, which is somewhat higher than our recently published values in the adult rat, which ranged from 3.6 to $4.3 \%$ (20). Small vessel plasma content of the rabbit brain has been reported to average $7.7 \%$ (21). Mean values of the number of capillaries (per $\mathrm{mm}^{2}$ ) in the present study were 279 to 374 in different brain regions, which was similar to the values of our study in the adult rat, which averaged 283 to 431 . The values for total surface area $\left(\mathrm{mm}^{2} / \mathrm{mm}^{3}\right)$ ranged from 18 to 21 in our study. These values were different from those reported in the newborn rat, for which volume fraction was approximately $0.5 \%$, increasing to $1.5 \%$ by $20 \mathrm{~d}$; surface area was approximately $4 \mathrm{~mm}^{2} / \mathrm{mm}^{3}$ at birth, increasing to 12 by $20 \mathrm{~d}$; and number was $170 / \mathrm{mm}^{2}$ at birth, increasing to 300 by $3 \mathrm{wk}(22,23)$. Newborn rat brain is quite immature at birth, whereas the newborn pig brain is quite mature; this may account for the difference in the various capillary morphometric measures. Potential regional cerebral metabolic differences have not been determined in the newborn pig. Chronic hypoxia in the first few weeks of life increases the length and the volume fraction of the total capillaries (22), whereas hypothyroidism retards the growth of brain capillaries in the neonatal period (23).

We were unable to fill $100 \%$ of the capillaries visualized with alkaline phosphatase with either of the perfusion markers. In the adult rat, if FITC-dextran is allowed to circulate for 6 min, over $95 \%$ of the capillaries can be filled with FITC-dextran. Devel- opment of brain capillaries in the newborn rat has been studied by Bar (22), Caley and Maxwell (24), and David and Nathaniel (23). In the rat, the blood vessels develop in the first $10 \mathrm{~d}$ of life as solid cords of mesodermal elements that form a network of primordial vessels. A blood-filled patent lumen develops in these blood vessels during the second 10 -d period. Our finding of incomplete filling of the circulation with plasma markers may reflect the presence of budding capillaries that may have the alkaline phosphatase enzyme but not the full lumen or full twosided connections with the circulation. It is also possible that alkaline phosphatase staining in the newborn animal may stain some structure other than capillaries. This is less likely, because with silver methenamine stain we also found similar results.

The time course of filling of capillaries with FITC-dextran in our study indicated that the newborn pig is different from the adult rat. At $6 \mathrm{~s}$ after injection, $50-60 \%$ of the capillaries in various brain regions were filled with FITC-dextran, which increased to $80-85 \%$ at $12 \mathrm{~s}$ and then remained unchanged up to 6 min after injection. This may be related to differences in transit times through cerebral capillaries in the pig. It has been shown that the newborn pig has an intact blood-brain barrier that does not change significantly with acidosis (15). The blood-brain barrier is controlled by both the permeability and the perfused surface area of the capillary network. In the newborn pig, it appears that perfused cerebral capillary surface area is maximal, and increases in transport across the blood-brain barrier can only be achieved by changes in permeability. However, any condition that is likely to decrease the number of perfused capillaries in the tissue, and thereby increase the diffusion distance for oxygen in the tissue, could reduce transport across the barrier. This is likely to cause cell injury and death in areas farthest from the perfused capillaries. These conditions include hemorrhage, hypotension, reperfusion after ischemia, and infusion of vasopressors.

In conclusion, our data indicate that FITC-dextran and alkaline phosphatase are suitable markers to stain the perfused and total capillary network in the newborn pig brain. The newborn pig lacks a cerebral capillary reserve at rest. We speculate that any agent that decreases the number of perfused cerebral capillaries is likely to cause focal neuronal injury and necrosis without necessarily affecting the integrity of the whole brain. Further studies are necessary to address this issue.

\section{REFERENCES}

1. Miller DJ, Bell AB 1987 Cerebral blood flow variations with perfusion pressure and metabolism. In: Wood JH (ed) Cerebral Blood Flow: Physiological and Clinical Aspects. McGraw-Hill, New York, pp 119-130

2. Volpe JJ 1987 Hypoxic-ischemic encephalopathy: biochemical and physiological aspects. In: Neurology of the Newborn. WB Saunders, Philadelphia, pp $180-189$

3. Weiss HR, Buchweitz E, Murtha TJ, Auletta M 1982 Quantitative regional determination of morphometric indices of the total and perfused capillary network in the rat brain. Circ Res 51:494-503

4. Buckley NM 1986 Maturation of circulatory system in three mammalian models of human development. Comp Biochem Physiol 83A:1-7

5. Hudlicka O, Tyler KR 1986 Angiogenesis: The Growth of the Vascular System. Academic Press, London, pp 52-55

6. Trommer BL, Groothuis DR, Pasternak JF 1987 Quantitative analysis of cerebral vessels in the newborn puppy: the structure of germinal matrix vessels may predispose to hemorrhage. Pediatr Res 22:23-28

7. Yoshida Y, Ikuta F, Watabe K, Nagata T 1985 Developmental microvascular architecture of the rat cerebellar cortex. Anat Embryol 171:129-138

8. Francois-Dainville E, Buchweitz E, Weiss HR 1986 Effect of hypoxia on the percent of the arteriolar and capillary beds perfused in rat brain. $J$ Appl Physiol 60:280-288

9. Grover GJ, Francois-Dainville E, Buchweitz E, Weiss HR 1986 Effect of hemorrhage on regional morphometric indices of cerebral capillarity. $J$ Appl Physiol 61:1712-1719

10. Weibel ER 1979 Stereological Methods, Vol 1, Practical Methods for Biological Morphometry. Academic Press, London

11. Vorbrodt AW, Lossinsky AS, Wisniewski HM 1986 Localization of alkaline phosphatase activity in endothelia of developing and mature mouse bloodbrain barrier. Dev Neurosci 8:1-13

12. Kalaria RN, Harik SI 1987 Blood-brain barrier monoamine oxidase: enzyme characterization in cerebral microvessels and other tissues from six mammalian species, including human. J Neurochem 49:856-864 
13. Fuglsang A, Lomholt M, Gjedde A 1986 Blood-brain transfer of glucose and glucose analogs in newborn rats. $\mathrm{J}$ Neurochem 46:1417-1428

14. Cornford EM, Cornford ME 1986 Nutrient transport and the blood-brain barrier in developing animals. Fed Proc 45:2065-2072

15. Wagerle LC, Kumar SP, Belik J, Delivoria-Papadopoulos M 1988 Blood-brain barrier to hydrogen ion during acute metabolic acidosis in piglets. $\mathbf{J} \mathrm{Appl}$ Physiol 65:776-781

16. Weiss HR 1988 Measurement of cerebral capillary perfusion with a fluorescent label. Microvasc Res 36:172-180

17. Gobel U, Klein B, Schrock H, Kuschinsky W 1989 Lack of capillary recruitment in the brains of awake rats during hypercapnia. J Cereb Blood Flow Metab 9:491-499

18. Klein B, Kuschinsky W, Schrock H, Vetterlein F 1986 Interdependency of local capillary density, blood flow and metabolism in the rat brains. Am J Physiol 251:H1333-H1340
19. Gobel U, Theilen H, Kuschinsky W 1990 Congruence of total and perfused capillary network in rat brains. Circ Res 66:271-281

20. Anwar M, Kissen I, Weiss HR 1990 Effect of chemodenervation on the cerebral vascular and microvascular response to hypoxia. Circ Res 67:1365-1373

21. Weiss HR, Edelman NH 1976 Effect of hypoxia on small vessel blood content of rabbit brain. Microvasc Res 12:305-315

22. Bar T 1980 The Vascular System of the Cerebral Cortex. Springer-Verlag, Berlin

23. David S, Nathaniel EJH 1981 Development of brain capillaries in euthyroid and hypothyroid rats. Exp Neurol 73:243-253

24. Caley DW, Maxwell DS 1970 Development of the blood vessels and extracellular spaces during postnatal maturation of rat cerebral cortex. $\mathbf{J}$ Comp Neurol 138:31-48

\section{Announcement}

\section{0th Annual Seminar in Pediatric Nephrology}

The 20th Annual Seminar in Pediatric Nephrology: Current Concepts in Diagnosis and Management will be held February 6-10, 1993 at the Fountainebleu Hilton Resort and Spa, Miami Beach, FL. Please contact Pearl Seidler at the University of Miami School of Medicine, Department of Pediatrics, PO Box 016960, Miami, FL 33101, (305) 585-6726, FAX (305) 547-1709. 